\title{
Testing homogeneity in Weibull error in variables models
}

\author{
Dione M. Valença • Heleno Bolfarine
}

Published online: 15 November 2006

(C) The Institute of Statistical Mathematics, Tokyo 2006

\section{Erratum to: AISM 58: 115-129 DOI 10.1007/s10463-005-0006-8}

The original version of the history unfortunately contained a mistake. The correct approval history is given here.

Received: 19 December 2003 / Revised: 29 November 2004

The online version of the original article can be found at http://dx.doi.org/10.1007/s10463-005-0006-8.

D. M. Valença

Departamento de Estatística, Universidade Federal do Rio Grande do Norte, CCET. CEP 59072-970, Natal, R.N., Brazil

e-mail: dione@ccet.ufrn.br

H. Bolfarine ( $\bowtie)$

Departamento de Estatística, Universidade de São Paulo, IME. Caixa Postal 66281, CEP 05311-970, São Paulo, S.P., Brazil

e-mail: hbolfar@ime.usp.br 